\title{
Gewinner des Fachdental Leipzig-Awards stehen fest
}

Weltneuheit, Exklusiv, Premiere - mit diesen Hinweisen präsentierten sich zahlreiche Aussteller am 23./24. September 2016 auf der Fachdental Leipzig. Über 4000 Besucher erhielten einen Überblick über aktuelle Produkte und wichtigste Innovationen der Dentalindustrie und des Handels. Neben den aktuellen Produkten an den Ständen stellten 5 Unternehmen ihre Neuprodukte exklusiv auf dem Marktplatz der Ideen vor und nominierten sich für den Fachdental Leipzig-Award. Der 1. Award-Preis geht an das Unternehmen Ivoclar Vivadent $\mathrm{GmbH}$ für den Cervitec F-Schutzlack, der Fluoridierung und
Keimkontrolle in 1 Arbeitsschritt bietet. Mit dem 2. Preis wurde ultraDEX, als Alternative zu Chlorhexidin, von MegadentDeflogrip ausgezeichnet. Auch W\&H zeigte auf dem Marktplatz der Ideen eine Innovation in der Premium-Klasse von Ultradent und erhält hierfür den 3. Preis. Mit der integrierten W\&H Primera Advanced Air Technology wurde ein vielseitiges und leistungsstarkes Handstück mit modernen Mircomotor speziell für die HighSpeed-Präparation entwickelt.

Nach einer Pressemitteilung der Landesmesse Stuttgart GmbH, Stuttgart www.messe-stuttgart.de 